\title{
PATRÓN DE ACTIVIDAD DIARIO DE LA HORMIGA Camponotus sp. EN LOS BOTONES FLORALES DEL CACTUS Neoraimondia arequipensis subsp. roseiflora (Werdermann \& Backeberg) Ostolaza
}

\section{DAILY ACTIVITY PATTERN OF THE Camponotus sp. ANT ON THE FLOWERING BUTTONS OF THE Neoraimondia arequipensis subsp. roseiflora (Werdermann \& Backeberg) Ostolaza CACTUS}

\author{
Sidney Novoa ${ }^{1}$, Inés Redolfi ${ }^{2}$ y Aldo Ceroni ${ }^{1}$
}

\begin{abstract}
Resumen
Con el propósito de monitorear la actividad diaria de la hormiga Camponotus sp. sobre el cactus Neoraimondia arequipensis subsp. roseiflora (Werdermann \& Backeberg) Ostolaza se realizó un estudio en el cerro Umarcata, cuenca baja del río Chillón, Lima, Perú. Las tres visitas al lugar fueron en los meses de Setiembre del 2003, Enero y Agosto del 2004. Se realizó un conteo de las obreras cada hora (durante 24 horas) en el instante en el que el observador fijaba su vista en el botón floral ( $\mathrm{n}=7$, en botones en cada una de las 10 plantas). Los resultados muestran que el patrón de actividad de Camponotus sp. en los tres momentos de visita fue unimodal, con un máximo entre las 6 y $7 \mathrm{pm}$ y un mínimo entre las 6 y 7 am. Igualmente Camponotus sp. presentó un mayor dinamismo durante las horas de la noche. La actividad diaria de la hormiga no se encuentra relacionada con la temperatura y la humedad relativa y estaría más ligada al fotoperiodo y a la oferta de alimento del cactus, el cual podría estar alcanzando sus picos de producción de néctar más altos durante la noche al igual que muchos otros cactus de floración nocturna.

Palabras clave: Camponotus sp., ecosistema árido, hormiga-cactácea, Neoraimondia arequipensis subsp. roseiflora, patrón de actividad diario.
\end{abstract}

\begin{abstract}
Monitoring of the daily activity of the Camponotus sp. ant over the Neoraimondia arequipensis subsp. roseiflora (Werdermann \& Backeberg) Ostolaza cactus was investigated at Umarcata hills, lower Chillon river basin, Lima, Peru. There were three visits to this site: on September 2003, January and August 2004. In each visit, hourly, for a 24-hour period, the number of working-ants on the button surface on the moment that the observer set his or her eyes on the floral button $(\mathrm{n}=7$ buttons on each of 10 plants) was registered. Results show that the activity pattern of Camponotus sp. on these three visits was unimodal, with a maximum between 6 and $7 \mathrm{pm}$, and a minimum between 6 and 7 am. Similarly, Camponotus sp. presented greater dynamism during night hours. Ant daily activity is not related to temperature, nor relative humidity, but it might be related to photoperiod and the cactus-food offer, which might be reaching its highest nectar production peak during the night, similarly to many other nocturnal flowering cacti.
\end{abstract}

Keywords: Camponotus sp., arid ecosystem, ant-cactaceae, Neoraimondia arequipensis subsp. roseiflora, daily activity pattern.

\section{Introducción}

Los formícidos se encuentran entre los organismos que dominan la tierra, con una biomasa del entorno del 10 al $15 \%$ del total de la biomasa animal en la mayoría de los ecosistemas (Hölldobler \& Wilson, 1990). El lugar que ocupan en los ecosistemas es muy importante, interviniendo en el ciclo de nutrientes en la naturaleza, en el enriquecimiento de los suelos y en una gran diversidad de interacciones tróficas, tanto que se considera que el flujo de energía que pasa a través de ellas es superior al que pasa a través de los vertebrados endotérmicos que viven en el mismo hábitat (Jaisson, 2000; Redolfi et al., 2003). El cactus Neoraimondia arequipensis subsp. roseiflora constituye el eje de los procesos aéreos en el ecosistema árido del valle del río Chillón. En este lugar, se destaca la importancia de la relación significativa de una especie de hormiga (Camponotus sp.) forrajeando el néctar que secretan hacia el exterior los botones florales del cactus $N$. arequipensis subsp. roseiflora, en una asociación entre el número de botones florales producidos y el número de hormigas visitantes. La visita de las hormigas presenta un pico máximo en el mes de Abril, en respuesta al estadio fenológico, a la temperatura y humedad relativa. El comportamiento agresivo por parte de Camponotus sp. frente a insectos fitófagos (Diptera y Lepidoptera), como la persecución de estos hasta su alejamiento del 
botón o flor, son un determinante de beneficio para el cactus. El néctar segregado podría ser la recompensa que reciben las hormigas por la protección frente a los herbívoros (Novoa et al., 2003, 2005 a b). Considerando la importancia de esta relación, el presente trabajo tiene la finalidad de profundizar en el conocimiento de la misma y determinar el patrón de actividad diario de la hormiga en los botones florales del cactus.

\section{Materiales y Métodos \\ Ubicación}

El área de la investigación se ubica en el cerro Umarcata, el cual se encuentra a una distancia de 6 kilómetros de Santa Rosa de Quives, a la altura del kilómetro 69 de la carretera Lima - Canta, a una altitud de 1260 msnm a 11'37'39', L.S. y 7646'9', L.O. en el valle del río Chillón, Departamento de Lima, en la Provincia de Canta (Novoa et al., 2003, 2005).

Determinación del patrón de actividad diario de la hormiga Camponotus sp. Se realizaron tres visitas al lugar en los meses de Setiembre del 2003, Enero y Agosto del 2004 con el propósito de monitorear la actividad diaria de la hormiga en diferentes momentos del año. En cada visita se evaluó durante 24 horas continuas la actividad de la hormiga sobre $N$. arequipensis subsp. roseiflora mediante el conteo de las mismas cada hora, en el instante en el que el observador fijaba su vista en el botón floral. Para esto se marcaron 7 botones o pimpollos florales elegidos al azar en cada una de las 10 plantas adultas (estas cantidades fueron escogidas en proporción al esfuerzo por búsqueda de botón). Paralelamente a estas evaluaciones se tomaron datos de temperatura y humedad relativa con un termohidrómetro digital.

\section{Resultados y Discusión}

Los resultados muestran que el patrón de actividad diario de Camponotus sp. en los tres momentos de visita fue unimodal, con un máximo entre las 6 y 7 pm y un mínimo entre las 6 y 7 am. Igualmente Camponotus sp. presentó un mayor dinamismo durante las horas de la noche al igual que otras hormigas de ecosistemas áridos (Pickett \& Clark, 1979). Aunque en la evaluación de Enero las temperaturas fueron más bajas de lo normal además de que llegó a precipitar, la hormiga Camponotus sp. no cambió su patrón de actividad aunque la proporción de hormigas por botón descendió (Figuras 1, 2 y 3). Por otra parte, la temperatura y la humedad no se encuentran relacionadas con la actividad diaria de la hormiga (temperatura: Setiembre $\mathrm{R}^{2}=0.0013$, Enero $\mathrm{R}^{2}=0.0047$, Agosto $\mathrm{R}^{2}=0.002$; humedad relativa: Setiembre $\mathrm{R}^{2}=0.0035$, Enero $\mathrm{R}^{2}=0.0011$, Agosto $\mathrm{R}^{2}=0.151$ ) (Figuras 4, 5 y 6) lo que muestra que dicho comportamiento estaría más ligado al fotoperiodo y a la oferta de alimento del cactus, el cual podría estar alcanzando sus picos de producción de néctar más altos durante la noche al igual que muchos otros cactus de floración nocturna (Valiente et al., 1997; Holland \& Fleming, 1999; De Viana et al., 2001; Clark \& Molina, 2004; Molina et al., 2004). Sin embargo, el número de hormigas visitantes se redujo en el mes de Enero en el que las temperaturas descendieron hasta $\operatorname{los} 18^{\circ} \mathrm{C}$ durante las horas de evaluación y la humedad relativa se incrementó llegando incluso hasta precipitar. Por lo que concluimos que la temperatura y la humedad relativa no afecta el patrón de actividad diario de la hormiga, pero si pueden ser factores que influyan en el número de hormigas que suele visitar normalmente al cactus (Figura 7). El patrón de actividad de las hormigas depende principalmente de la permanencia del recurso alimenticio de su preferencia en el medio y los rangos óptimos de las diferentes variables ambientales (Cerdá \& Retana, 1988; Cerdá et al., 1989; Farji-Brener, 1993; Morris, 1997; Redolfi et al., 2003). En observaciones adicionales, se constató el tiempo de la apertura floral desde las $6 \mathrm{pm}$ hasta las $7 \mathrm{am}$, horas que coinciden con la llegada de algunos visitantes florales como las polillas de la familia Pyralidae que también consumen los azúcares que secretan los botones florales pero que son rápidamente ahuyentadas por la hormiga.

\section{Agradecimientos}

Los autores expresan su agradecimiento a los estudiantes de Biología de la UNALM que colaboraron en la toma de datos. Esta investigación fue financiada por el Fondo Especial de Desarrollo Universitario (FEDU) de la Universidad Nacional Agraria La Molina.

\section{Literatura citada}

Cerdá X. \& Retana J.1988. Descripción de la comunidad de hormigas de un prado sabanoide en Canet de Mar (Barcelona). Ecología. 2: 333-341.

Cerdá X., Retana J., Bosch J. \& Alsina A. 1989. Daily foraging activity and food collection of the thermophilic ant Cataglyphis cursor (Hym. Formicidae). Vie Milleu. 39: 207-212.

Clark R. \& Molina F. 2004. Reproductive ecology of the rare clonal cactus Stenocereus eruca in the Sonoran deseret. Plant Systematics and Evolution. 247(3/4): 155164.

De Viana M., Ortega B., Saravia M. \& Schlumpberger B. 2001. Biología floral y polinizadores de Trichocereus pasacana (Cactaceae) en el Parque Nacional Los Cardones, Argentina. Revista de Biología Tropical. 49(1): 279-285.

Farji-Brener A.G. 1993 Influencia de la estacionalidad sobre los ritmos forrajeros de Atta laevigata (Hymenoptera: Formicidae) en una sabana tropical. Revista de Biología Tropical. 41 (3): 897-899.

Holland J. \& Fleming T. 1999. Mutualistic interactions between Upiga virescens (Pyralidae), a pollinating seedconsumer, and Lophocereus schottii (Cactaceae). Ecology. 80(6): 2074-2084. 
Holldobler B. \& Wilson E.O. 1990. The Ants. Belknap Press of Harvard University, Cambridge, Massachusetts.

Jaisson P. 2000. La Hormiga y el Sociobiólogo. Fondo de Cultura Económica. 1ra. Ed. México.

Molina F., Rojas A., Fleming T. \& Valiente A. 2004. Pollination biology of the columnar cactus Pachycereus pecten-aboriginum in north-western México. Journal of Arid Environments. 56: 117-127.

Morris T.I. 1997. Interrelaciones entre olivos, plagas y depredadores. Tesis Doctoral. Universidad de Granada.

Novoa S., Castro V., Ceroni A. \& Redolfi I. 2003. Relación entre la hormiga Camponotus sp. (Hymenoptera: Formicidae) y una comunidad de cactus (Cactaceae) en el valle del río Chillón. Ecol. apl. 2 (1): 69-73.

Novoa S., Ceroni A. \& Arellano C. 2005 a. Contribución al conocimiento de la fenología del cactus Neoraimondia arequipensis subsp. roseiflora (Werdermann \& Backeberg) Ostolaza (Cactaceae) en el valle del río Chillón, Lima-Perú. Ecol. apl. 4 (1/2) (en prensa).
Novoa S., Redolfi I., Ceroni A. \& Arellano C. 2005 b. El forrageo de la hormiga Camponotus sp. en los botones florales del cactus Neoraimondia arequipensis subsp. roseiflora (Werdermann \& Backeberg) Ostolaza (Cactaceae). Ecol. apl. 4 (1/2) (en prensa).

Pickett C. \& Clark W. 1979. The function of extrafloral nectarines in Opuntia hocarpa (Cactaceae). American Journal of Botany. 66: 618-625.

Redolfi I., Tinaut A., Pascual F. \& Campos M. 2003. Patrón de actividad de Tapinoma nigerrima Nylander y Crematogaster scutellaris Olivier (Hymenoptera: Formicidae) en el cultivo de olivo y en el laboratorio. Zoológica Baetica. 13/14: 37-55.

Valiente A., Rojas A., Del Coro M. \& Davila P. 1997. Pollination Biology of two columnar cacti (Neubuxbaumia mezcalaensis and Neobuxbaumia macrocephala) in the Tehuacan Valley, central Mexico. American Journal of Botany. 84(4): 452-455.

\section{Figuras citadas en el texto:}
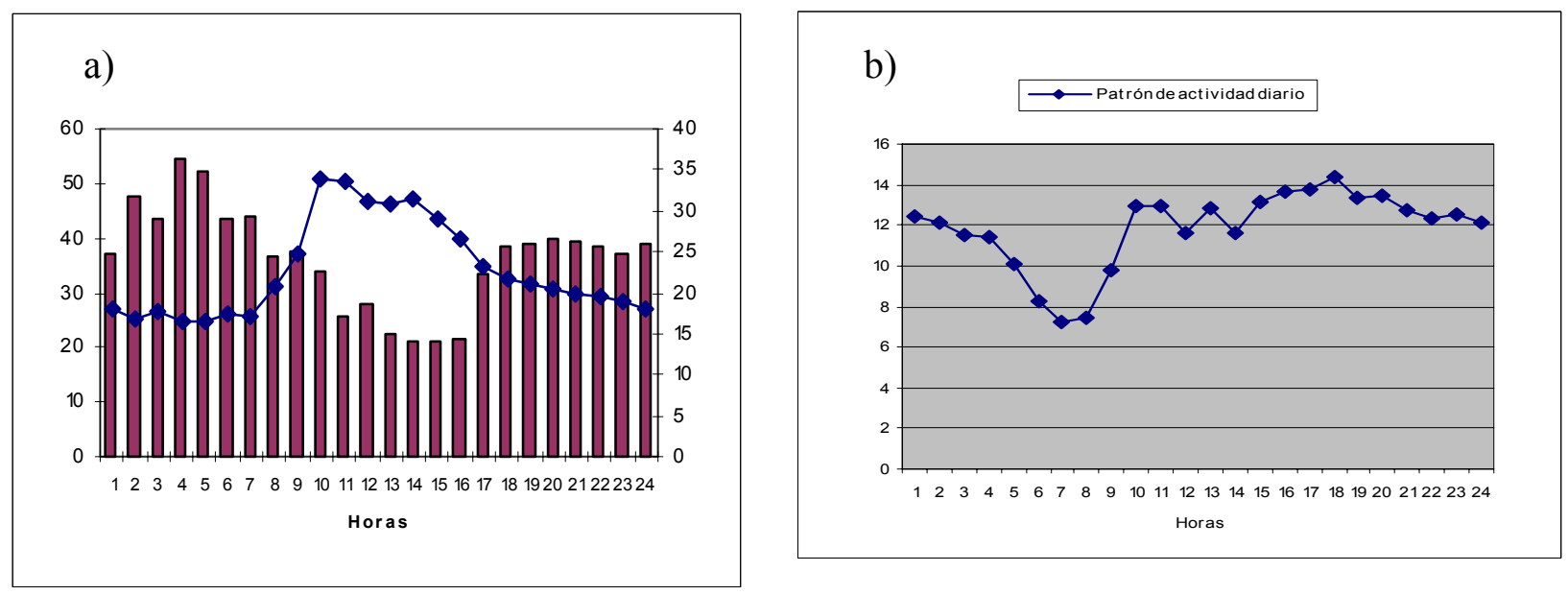

Figura 1. Variación de la temperatura $(-\bullet)$ y humedad relativa $(\square)$ en el cerro Umarcata, cuenca baja del río Chillón (a) y patrón de actividad diario de la hormiga Camponotus sp. sobre $N$. arequipensis subsp. roseiflora (b). 20 de Setiembre 2003. 

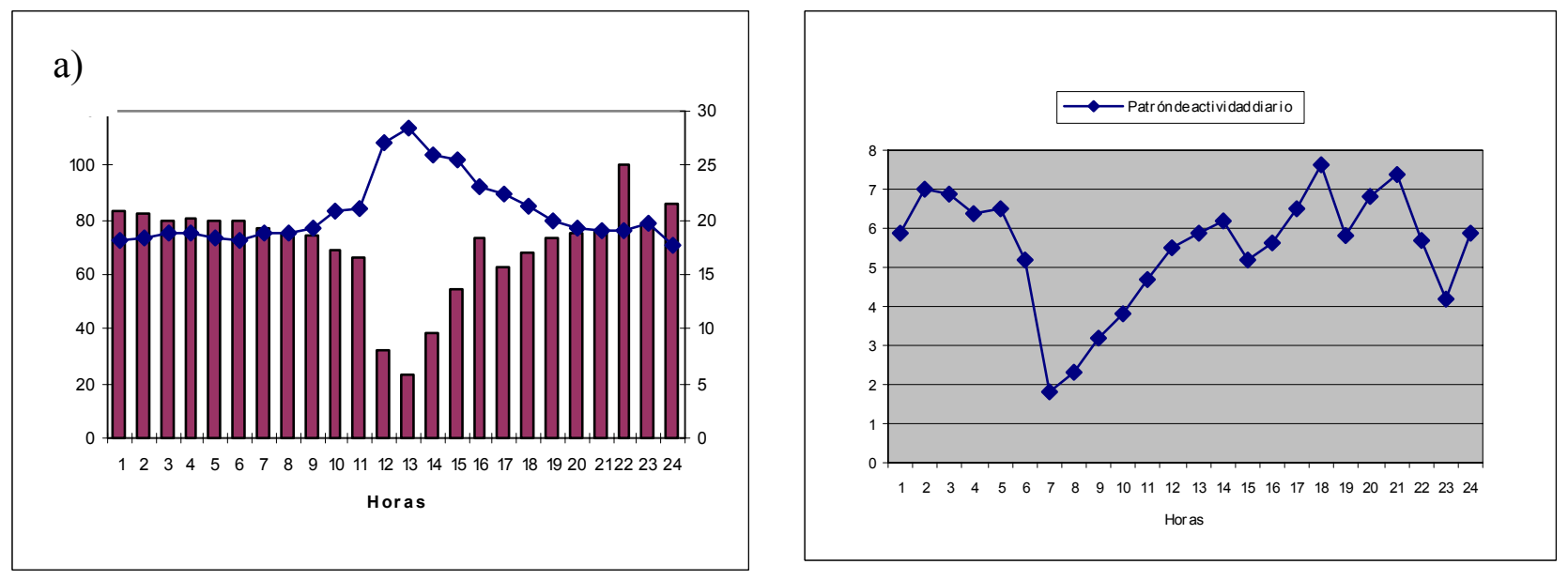

Figura 2. Variación de la temperatura $(\multimap-$ y humedad relativa $(\square)$ en el cerro Umarcata, cuenca baja del río Chillón (a) y patrón de actividad diario de la hormiga Camponotus sp. sobre $N$. arequipensis subsp. roseiflora (b). 31 de Enero 2004.
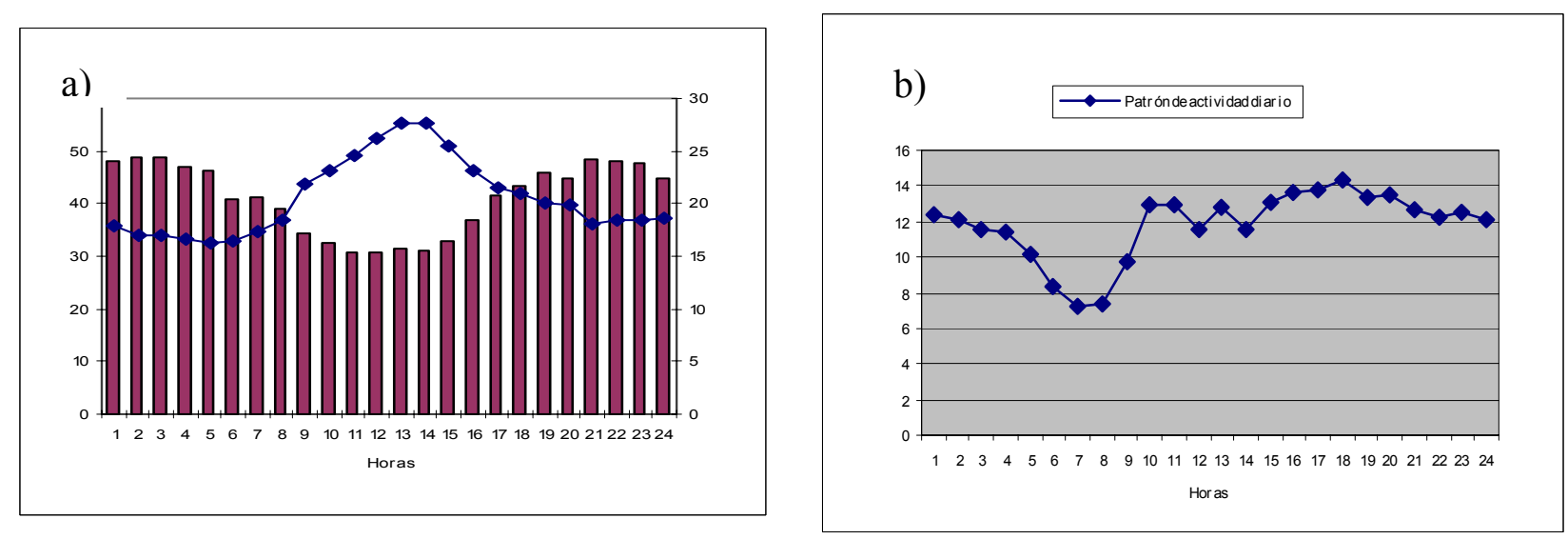

Figura 3. Variación de la temperatura $(\rightarrow)$ y humedad relativa $(\square)$ en el cerro Umarcata, cuenca baja del río Chillón (a) y patrón de actividad diario de la hormiga Camponotus sp. sobre N. arequipensis subsp. roseiflora (b). 20 de Agosto 2004.
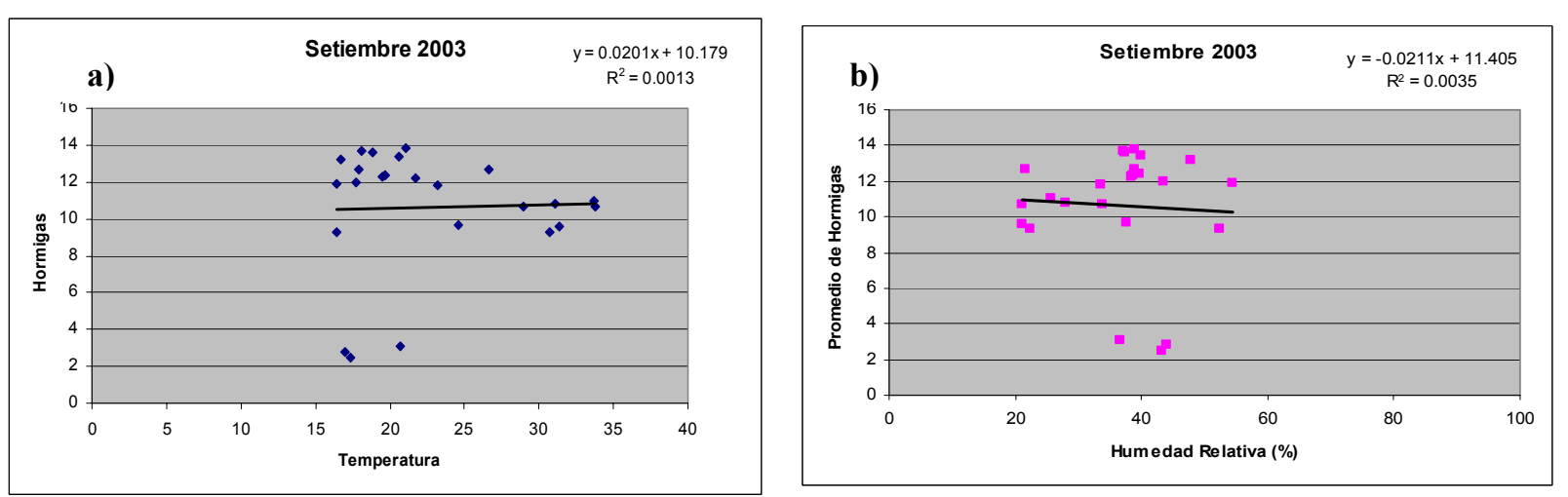

Figura 4. Relación entre la temperatura (a) y la humedad relativa (b) con la actividad de la hormiga Camponotus sp. sobre los botones florales. 20 de Setiembre 2003. 

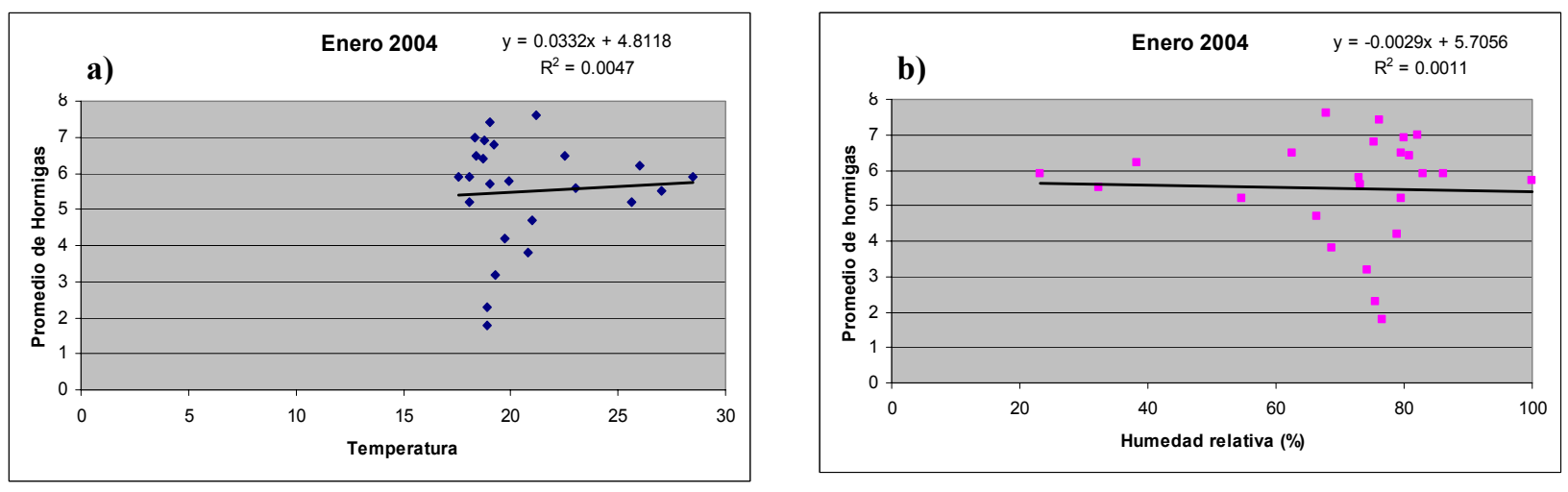

Figura 5. Relación entre la temperatura (a) y la humedad relativa (b) con la actividad de la hormiga Camponotus sp. sobre los botones florales. 31 de Enero 2004.
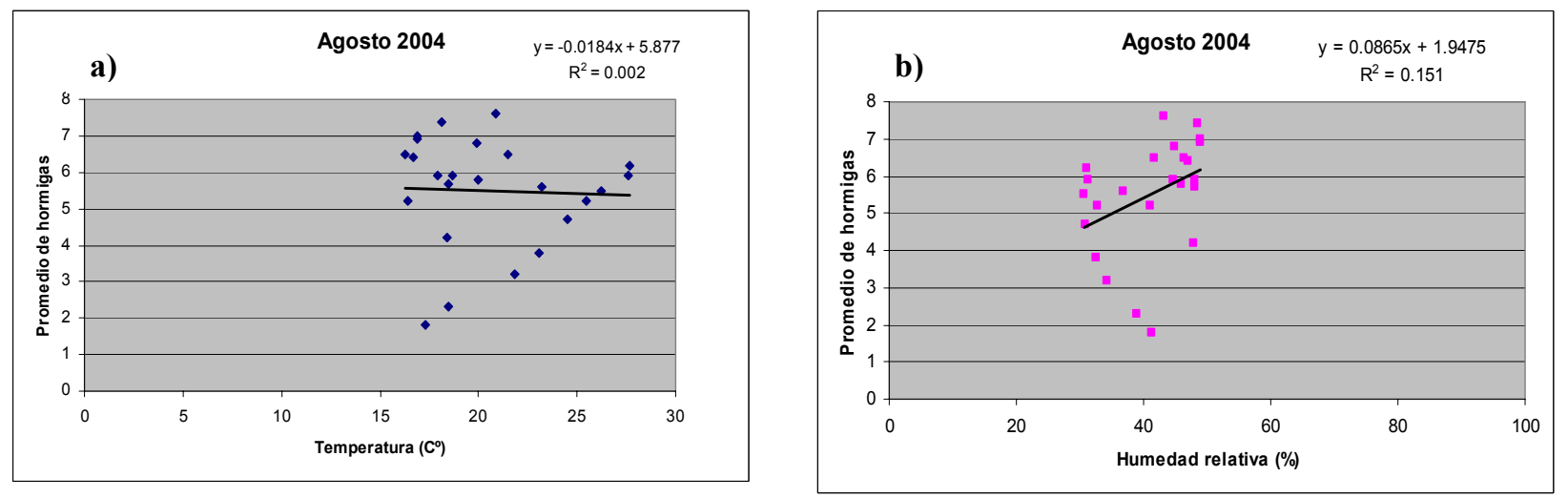

Figura 6. Relación entre la temperatura (a) y la humedad relativa (b) con la actividad de la hormiga Camponotus sp. sobre los botones florales. 20 de Agosto 2004.

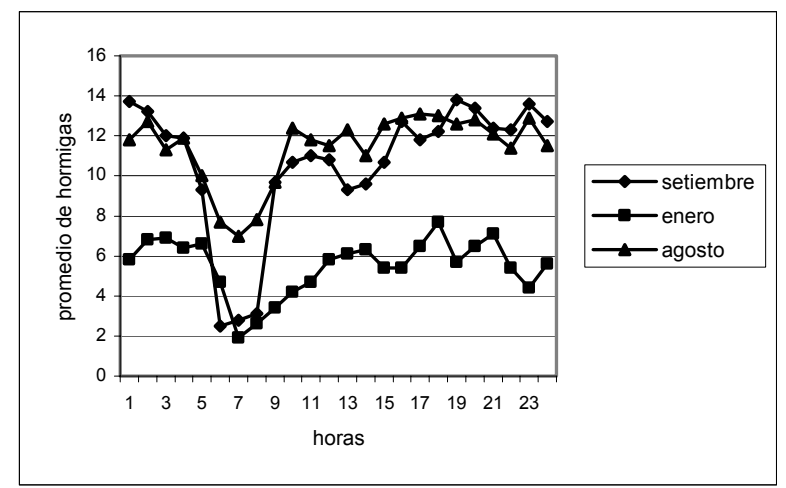

Figura 7. Patrón de actividad de la hormiga Camponotus sp. sobre N. arequipensis subsp. roseiflora durante los días 20 de Setiembre del 2003, y 31 de Enero y 20 de Agosto del 2004.

\footnotetext{
"Jardín Botánico “Octavio Velarde Núñez”. Departamento Académico de Biología. Universidad Nacional Agraria La Molina. Av. La Molina s/n, Apartado Postal 12056, Lima100. Perú, 2000084@lamolina.edu.pe, aceroni@lamolina.edu.pe

${ }^{2}$ Laboratorio de Control Biológico y Ecología de Artrópodos. Departamento Académico de Biología, Universidad Nacional Agraria La Molina, rhi@lamolina.edu.pe
} 